\title{
Screening bei jedem Sechsten falsch-positiv
}

Bei einem von sechs Männern, die am Prostatakrebs-Screening teilnehmen, ist mit einem erhöhten PSA-Wert ohne Befund in der Biopsie zu rechnen. Häufig haben diese Männer gleich mehrfach falsch-positive Befunde. Ihr Risiko für eine Prostatakarzinom-Diagnose ist allerdings auch erhöht.

F ür die Untersuchung wurden Daten von 61.604 Männern ausgewertet, die in Belgien, Finnland, Italien, den Niederlanden oder Schweden am Screening teilgenommen hatten. Je nach Land wurden die Männer alle zwei bis sieben Jahre einbestellt, der PSA-Grenzwert lag zwischen 3,0 und $4,0 \mathrm{ng} / \mathrm{ml}$. Ein höherer Wert, der nicht binnen eines Jahres histologisch auf ein Prostatakarzinom zurückgeführt wurde, galt als falsch-positiver Befund.

Mithilfe der Screening-Untersuchungen wurden 4.733 Prostatakarzinome entdeckt. Die Wahrscheinlichkeit einer Diagnose betrug 3,4\% in der ersten und zweiten und 3,6\% in der dritten Screening-Runde. Der Anteil falsch-positiver Ergebnisse lag bei 10,2, 11,0 und 11,1\%. Mit dem Alter der Getesteten nahm nicht nur die Zahl der Krebsdiagnosen, sondern auch die der falsch-positiven Befunde zu.

$17,8 \%$ der Männer erhielten aufgrund ihres PSA-Wertes mindestens einmal eine falsche Verdachtsdiagnose. Bei jedem vierten dieser Männer war das sogar zwei- oder dreimal der Fall. Etwa ein Viertel der einmal falsch-positiv getesteten Männer lehnte weitere Screening-Untersuchungen ab. Von denen, die ihre Teilnahme fortsetzten, hatten ungefähr $50 \%$ in der nächsten Runde wieder einen falsch-positiven Befund. Bei etwa $10 \%$ wurde mithilfe des Screenings doch noch ein Karzinom entdeckt; damit war das Risiko etwa viermal so hoch wie bei den zuvor negativ getesteten Männern.

Bei den falsch-positiven Ergebnissen gab es erhebliche Differenzen zwischen den Ländern: Italien hatte mit 11,1 \% die niedrigste und die Niederlande hatten mit $26,4 \%$ die höchste Rate, vermutlich wegen der niedrigeren PSA-Grenzwerte, die in Belgien, den Niederlanden und Schweden bei $3,0 \mathrm{ng} / \mathrm{ml}$, in Finnland und Italien aber höher liegen. Hätten alle Zentren einen Grenzwert von 4,0 ng/ml verwendet, so die Berechnung der Studienautoren, wären nicht $17,8 \%$, sondern nur $11,7 \%$ der Männer von falsch-positiven Befunden betroffen gewesen. Dafür wären allerdings

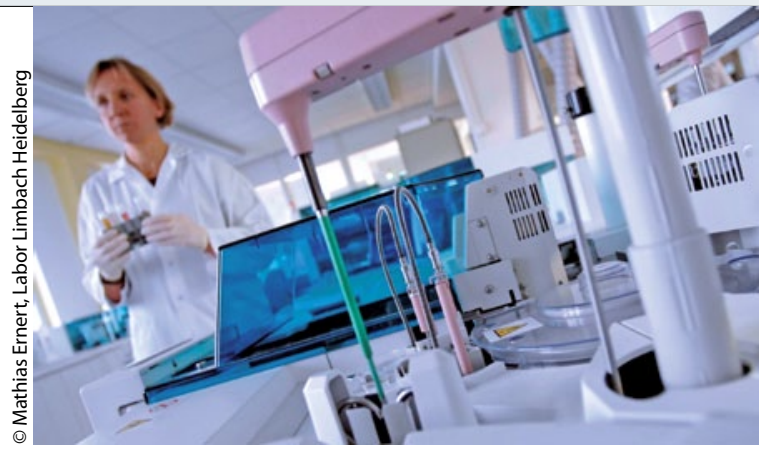

Ist der PSA-Test einmal falsch-positiv, ist das oft auch beim nächsten Mal der Fall.

auch 1.252 Prostatakarzinome übersehen worden. Beate Schumacher

Kilpeläinen TP et al. False-positive screening results in the European randomized study of screening for prostate cancer. Eur J Cancer. 2011;47(18):2698-705.

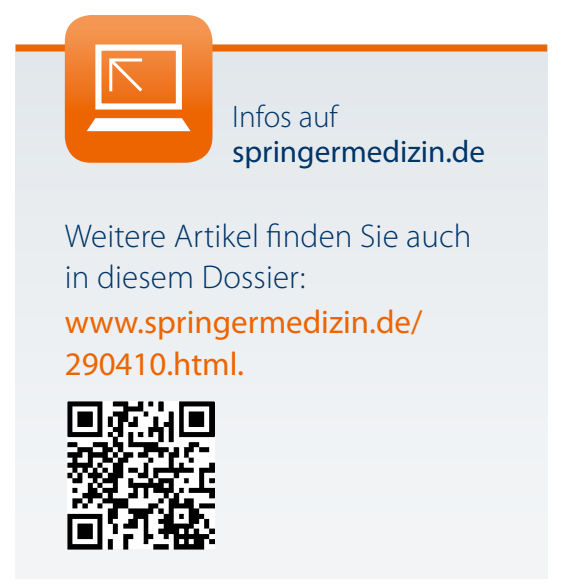

\section{BRCA1-Mutationen kein Risikofaktor}

Welchen Einfluss haben Mutationen des BRCA1-Gens beim Prostatakarzinom? In einer Fall-Kontroll-Studie wurde dies am Beispiel einer galizischen FounderMutation $\mathrm{G}$ und in einer Metaanalyse aller zugänglichen BRCA1-Genotypisierungsstudien untersucht.

O b Keimbahnmutationen des BRCA1Gens für ein Prostatakarzinom prädisponieren, ist bisher aufgrund widersprüchlicher Ergebnisse nicht eindeutig geklärt, zumal die geringen Fallzahlen einschlägiger Studien die statistische Power einschränken. An der Universitätsklinik von Santiago de Compostela, Spanien wurde deshalb eine Fall-Kontroll-Studie mit 905 unselektierten Prostatakarzinom-Patienten und 936 nicht verwandten, gesunden Probanden durchgeführt. Sie sollte Aufschluss über eine mögliche Rolle der galizischen Founder-Mutation c.211A >G liefern, die bei über $50 \%$ der galizischen Risikofamilien für BRCA1-assoziierten Brust-/Ovarialkrebs nachweisbar ist. Zusätzlich wurde in einer Metaanalyse von Literaturdaten der Effekt von BRCA1Mutationen auf die Entwicklung eines Prostatakarzinoms untersucht.

In der Genanalyse wurde bei einem Mann mit Prostatakrebs und bei drei gesunden Kontrollen die Mutation gefunden (Odds Ratio [OR] 0,27; 95\%-Konfidenzintervall [95\%-KI] 0,01-2,36; $\mathrm{p}=0,28$ ). Eine Metaanalyse dieser Daten zusammen mit den Angaben aus sieben weiteren
BRCA1-Genotypisierungsstudien (5.705 Prostatakarzinom-Patienten und 13.218 Kontrollen) ergab ebenfalls keine direkte Assoziation zwischen dem Risiko für ein Prostatakarzinom und dem Vorliegen einer BRCA1-Mutation (OR 1,36; 95\%-KI $0,87-2,14 ; \mathrm{p}=0,18$ ).

Fazit: Weder in einer monozentrischen Fall-Kontroll-Studie noch in einer Metaanalyse von sieben weiteren BRCA-1Genotypisierungs-Studien lieferte Hinweise auf eine Assoziation zwischen BRCA1-Mutationen und dem Risiko für ein klinisch manifestes Prostatakarzinom.

Barbara Kreutzkamp

Fachal L et al. BRCA1 mutations do not increase prostate cancer risk: results from a meta-analysis including new data.

Prostate. 2011;71(16):1768-79. 